\title{
Pragmática textual en adolescentes con trastorno por déficitit de atención/hiperactividad: argumentación
}

\author{
Beatriz Gallardo-Paúls, Manuel Gimeno Martínez, Verónica Moreno Campos
}

Introducción. La lingüística clínica asume un estudio del déficit lingüístico cuyo foco de atención se desplaza desde los aspectos estrictamente formales, gramaticales, al uso eficaz y contextualizado del lenguaje. En este sentido, resulta también inevitable la preocupación por el correlato cognitivo, mental, de dicho uso lingüístico, cuyas dimensiones textuales básicas son la narración y la argumentación.

Objetivo. Descripción de las habilidades argumentativas en adolescentes con trastorno por déficit de atención/hiperactividad (TDAH), atendiendo a su relación con el rendimiento académico y la sociabilidad.

Materiales y métodos. Analizamos 79 textos argumentativos de adolescentes con TDAH, con una metodología propia de la lingüística cognitiva y de las teorías de la argumentación de base dialógica.

Resultados y conclusiones. Los adolescentes con TDAH proporcionan mayor número de argumentos que los del grupo control, pero con un mayor predominio de estrategias emocionales y de sanción negativa, frente a un mayor uso de argumentos falaces o circulares en los del grupo control; no es significativa la diferencia en el uso de argumentos racionales en ambos grupos.

Palabras clave. Déficit lingüístico. Pragmática textual. TDAH.

\section{Introducción y objetivos}

En las últimas décadas, los estudios sobre las habilidades lingüísticas en poblaciones con daño neurológico se han visto claramente marcadas por el sesgo pragmático, que desplaza el foco de atención desde los aspectos estrictamente formales, gramaticales, al uso eficaz y contextualizado del lenguaje. En este sentido, resulta también inevitable la preocupación por el correlato cognitivo de dicho uso lingüístico, cuyas dimensiones textuales básicas son la narración y la argumentación [1-3]. Nuestro objetivo es profundizar en las habilidades argumentativas desarrolladas por niños con trastorno por déficit de atención/hiperactividad (TDAH) en la etapa adolescente, vinculando tales capacidades con dos aspectos importantes asociados al TDAH: el rendimiento académico y la sociabilidad inter pares.

\section{Antecedentes: pragmática textual en niños con TDAH}

En trabajos anteriores [4] hemos descrito las habilidades pragmáticas de un grupo de niños y niñas de edad escolar (9-11 años), a los que habíamos pedido la elaboración de textos narrativos y argumenta- tivos. En aquella investigación analizamos aspectos de densidad textual, como la proporción de sucesos $\mathrm{y}$ argumentos en cada texto, o las huellas formales explícitas de capacidad intersubjetiva (teoría de la mente).

Se comprobó que los textos escritos por niños y niñas con TDAH presentaban medidas levemente inferiores que el grupo control (especialmente en las franjas de menor edad) en algunos aspectos; por ejemplo, introducían más sucesos incoherentes y aducían menos justificaciones (una media de 3,3 argumentos por texto, frente a 5,2 en el grupo control). Se trataba, en definitiva, de textos menos elaborados y menos complejos que los redactados por sus pares. En cuanto al uso de diferentes tipos argumentativos, se comprobó que ambos grupos recurrían a las cuatro estrategias fundamentales identificadas por la teoría de la argumentación:

- Estrategias lógicas o de instrucción. Representan un $28,2 \%$ de los argumentos usados por el grupo con TDAH y un $34,8 \%$ en el grupo control; incluimos aquí las argumentaciones referidas a consecuencias más o menos objetivas, y también a las justificaciones realizadas mediante ejemplos: 'es como si tú le pegaras a él y él no te ha hecho nada'. Son argumentos que apelan a la razón, al juicio fundamentado (se excluyen las ejemplificaciones falaces, no pertinentes).
Facultad de Filología; Universitat de València; Valencia (B. Gallardo-Paúls, V. MorenoCampos). IES La Nucia; Alicante, España (M. Gimeno-Martínez).

Correspondencia: Dra. Beatriz Gallardo Paúls. Departamento de Lingüística General. Facultad de Filología. Avda. Blasco Ibáñez, 32, 5.‥ E-46010 Valencia.

E-mail: beatriz.gallardo@uv.es

Financiación:

Este trabajo se engloba en los proyectos 'Coherencia, cohesión y pragmática textual en situaciones de déficit lingüístico' (MICINN, FFI2008-02592/ FILO) y 'Protocolo de análisis pragmasintáctico. Un estudio de corpus oral' (MEC, HUM200766074-C02-02).

Aceptado: 24.12.09.

Cómo citar este artículo: Gallardo-Paúls B, GimenoMartínez M, Moreno-Campos V. Pragmática textual en adolescentes con trastorno por déficit de atención/ hiperactividad: argumentación. Rev Neurol 2010; 50: S113-7. 
- Estrategias argumentativas de sanción. Se basan en mecanismos de causa-efecto (de premio o castigo); son 69 argumentos en el total de la muestra, de los cuales sólo dos son de premio: 'no hay que pegar, porque si nos pegamos nos castigan y si son muchas veces nos pueden abrir un expediente'. Mientras en el grupo con TDAH constituyen un 33,3\% del total de argumentos, en el grupo control suponen un 17,7\%. En ambos casos tienen un notable valor ecoico respecto al discurso de autoridad que reciben los niños en su entorno.

- Estrategias altruistas o moralistas. Apelan a la identificación y la manipulación emotiva entre emisor y receptor: 'hay que respetar a los demás porque así los demás te respetan a ti'. Hemos incluido todos los argumentos que enmascaran falta de argumentos reales o revelan visiones catastrofistas, otra vez con claros matices ecoicos: 'si no hacemos unas ciertas normas nos podemos volver locos, sin normas todo esto parecería un zoo. Suponen un 28,2\% de los argumentos del grupo con TDAH (30,1\% en el grupo control).

- Estrategias claramente falaces. Incluimos tanto las de argumentación circular, tautológica, como las que remiten a topoi culturales, es decir, asunciones sancionadas socialmente y que configuran un nivel de vox populi donde la argumentación no se cuestiona.

\section{Materiales y métodos}

\section{Datos}

En la presente investigación hemos ampliado el rango de edad y presentamos un análisis similar referido a 79 textos de adolescentes con TDAH, cuya franja de edad se sitúa entre 12 y 18 años. Los textos han sido redactados por pacientes del Instituto Valenciano de Neurología Pediátrica (INVANEP) y por alumnos de institutos de enseñanza secundaria de Alicante y Valencia, que cuentan con el correspondiente diagnóstico de las Unidades de Salud Mental Infantil (USMI).

Centramos nuestro interés en el contraste que establecen tales datos con los procedentes de niños entre 7 y 11 años [4,5], y con 159 redacciones pertenecientes a un grupo control de adolescentes sin diagnóstico de TDAH de los mismos institutos [6]. Estos datos escritos forman parte de un corpus mayor en el que incluimos también datos argumentativos orales de adolescentes con y sin diagnóstico de TDAH en prácticas de debate y exposición oral.
La obtención de datos escritos se realizó mediante una prueba escrita, en el marco de asignaturas de lengua.

\section{Argumentación, adolescencia y TDAH}

La argumentación constituye, junto a la narración, una superestructura textual básica [1,2]; aunque el germen de ambos tipos puede rastrearse en la conducta verbal infantil (un llanto desconsolado puede ser un poderoso argumento), el esquema cognitivo que subyace a ambas estructuras textuales se consolida en momentos distintos. Cabe pensar que el ser humano estabiliza las categorías narrativas durante la etapa escolar, mientras que las categorías del esquema argumentativo (premisas o argumentos, garantías, ejemplos, datos, refutación, tesis o conclusión) se consolidan en épocas más tardías. Algunos autores $[7,8]$ han señalado que a los 3-4 años se empieza ya a proporcionar argumentos, pero que la estructura textual como tal surge sobre los 10-13 años y se consolida en torno a los 15-17 años, es decir, en plena adolescencia. Esta adquisición más tardía se debe, por un lado, a la mayor complejidad cognitiva de la argumentación y, por otro, al clarísimo predominio de los modelos narrativos sobre los argumentativos en nuestro entorno sociocultural (incluyendo el ámbito académico).

Los elementos fundamentales del texto argumentativo son una afirmación para la cual se propone un valor veritativo (la conclusión), y unos argumentos (premisas o justificaciones) que se proponen como aval o garantía de tal afirmación. Sin embargo, mientras existe una argumentación lógica basada en el valor veritativo de los enunciados ('Sócrates es humano, luego Sócrates es mortal', etc.), la argumentación cotidiana de las lenguas naturales se distingue por utilizar argumentos que no se basan sólo en la verdad o la razón, sino en la verosimilitud. Surgen así las distintas tipologías sobre argumentos utilizados, que ya hemos visto. Lo que nos interesa en este trabajo es que los tipos de argumentos remiten a estrategias cognitivo-persuasivas diferentes, las cuales pueden verse afectadas por la existencia de rasgos como hiperactividad, impulsividad o inatención.

La adolescencia es la etapa en que el individuo se debe forjar un universo de pensamiento propio y en la que las relaciones con los demás adquieren una dimensión fundamental. En este sentido, se suele señalar que los adolescentes con TDAH presentan una baja competencia social y de rendimiento intelectual [9-12]. El déficit de control inhibitorio y la impulsividad les hacen presentar escasas habilida- 
des sociales, lo que provoca problemas de relación inter pares: la relación con sus compañeros puede ser conflictiva, acostumbran a quedarse sin amigos y pueden ser rechazados.

Uno de los problemas derivados de la incapacidad inhibitoria es la violación de normas y reglas, de ahí que el paso por la escuela de los alumnos con TDAH sea conflictivo, porque les exige autocontrol y esfuerzo sostenido; los niños con TDAH presentan bajo rendimiento escolar y alto índice de repeticiones. Asimismo, son muy susceptibles de ser sancionados con expulsiones y, en ocasiones, con expedientes disciplinarios.

En el caso de los adolescentes con TDAH, la llegada al instituto propicia un empeoramiento de los resultados académicos, en buena parte porque deben enfrentarse a una serie de cambios: mayor número de profesores y asignaturas, horarios más condensados, compañeros nuevos, creciente énfasis en la autonomía del alumno, menos atención individualizada... En este contexto, se explica la existencia de lo que Barkley [13,14] etiqueta como formas de comunicación negativa en TDAH, y que podemos clasificar, según nuestro enfoque pragmático, en tres tipos de conducta:

- Actos de habla ilocutivos: insultar, menospreciar al otro, criticar continuamente, ponerse a la defensiva, salirse del tema, sacar a relucir temas del pasado, minimizar temas graves.

- Paralenguaje: evitar la mirada, usar tono sarcástico o desdeñoso.

- Conducta y psicología: tener rabietas, eludir responsabilidades, teoría de la mente inadecuada.

Casi todas estas conductas (lingüísticas o no), de impacto social negativo, afectan a la capacidad argumentativa, al igual que otras directamente vinculadas con los dos criterios básicos de identificación del trastorno [6]. Así, cabe pensar que la impulsividad provoque en los hablantes:

- Atolondramiento o precipitación argumentativa.

- Falta de previsión de la refutación del interlocutor.

- Dificultades para el silencio estratégico: indiscreción.

- Interpretaciones literales que no profundizan en las inferencias.

- Tendencia a interrumpir.

Por otro lado, la inatención puede originar disfunciones pragmáticas, como dificultad para manejar simultáneamente muchos argumentos y sus consecuencias, y problemas para escuchar atentamente.

A partir de la propuesta de Barkley, que explica el TDAH como un déficit de control inhibitorio, pode- mos prever problemas con los procesos generales de procesamiento de la información (análisis y síntesis) [15], ya que exigen una capacidad inhibitoria y de espera, cuya carencia en los hablantes con TDAH crea dificultades en aspectos como la toma de turno o la gestión temática, rasgos pragmáticos esenciales en algunos ejercicios argumentativos como los debates. Por otra parte, este mismo déficit inhibitorio podría explicar que los niños y adolescentes con TDAH sean más emotivos, en la medida en que su interpretación de los mensajes es más personal y subjetiva; esta dificultad para inhibir los sentimientos también les haría creer más en sus opiniones y, por tanto, ser más vehementes/convincentes.

Por último, cabría comentar los problemas que presentan los hablantes con TDAH en relación a la interiorización del lenguaje. Las autoverbalizaciones se asocian a la atención focalizada, de ahí que sean utilizadas por muchas propuestas de intervención cognitiva en TDAH [16]. Esta irregular interiorización del lenguaje influiría negativamente en procesos cognitivos imprescindibles en la argumentación como la autorreflexión, el autocuestionamiento y el razonamiento moral.

\section{Resultados y discusión}

El análisis de los datos muestra las siguientes tendencias en los textos de adolescentes con TDAH:

- La argumentación más utilizada es la falaz, y dentro de ella, la estrategia emotiva basada en la identificación con el receptor. Estos argumentos moralistas, altruistas, constituyen un $25,9 \%$ del total de los argumentos utilizados por nuestro grupo adolescente. Sin abandonar la esfera de la falacia, encontramos también un recurso frecuente $(17,1 \%$ del total de la muestra) a las argumentaciones circulares y tautológicas, así como a las que se basan en tópicos socialmente ratificados (10,9\%).

- Las estrategias de instrucción, basadas en argumentos lógicos y objetivos o en ejemplos ilustrativos, constituyen en total un 32,9\% de los argumentos utilizados (si bien sólo 17 de los 433 argumentos son ejemplos).

- Las estrategias de sanción suponen un 12,7\% del total de argumentos; de los 55 argumentos de sanción, sólo cinco aducen una estrategia de premio, mientras los otros 50 aluden a castigos y consecuencias negativas.

- Se ve una tendencia a la reiteración de un mismo argumento, que nos llevaría a pensar en una falta de flexibilidad cognitiva y escasa consideración a nuevos puntos de vista. 
Si intentamos establecer una evolución por referencia a los textos infantiles (Tabla I), vemos que los rasgos más notables son el ligero incremento de estrategias lógicas y lo que parece una sustitución de las argumentaciones basadas en el castigo por las argumentaciones circulares ('no se puede fumar en el instituto porque no se puede'), o aquellas que se basan en tópicos aceptados; de entre estas últimas es destacable el uso de tópicos relacionados con la salud y los denominados hedonistas.

Por lo que se refiere al contraste con las redacciones del grupo control, encontramos que, una vez más, no hay diferencias radicales entre uno y otro grupo (Tabla II). Destaca, en primer lugar, el hecho de que los adolescentes del grupo control proporcionan una media de 3 justificaciones por texto, frente a las 5,5 del grupo con TDAH. Por lo demás, vemos mayor proporción de argumentos basados en la sanción (de castigo): un 12,7\% en los adolescentes con TDAH frente a un 6,3\% de las argumentaciones usadas por el grupo control; este incremento no se compensa en el grupo control con un mayor recurso a estrategias lógicas, sino que se mantiene en el ámbito de la falacia (argumentos tópicos, tautológicos o de autoridad).

Y es que tanto los textos de informantes con TDAH como los del grupo control se mueven en un ámbito próximo a lo que Maliandi denomina 'pseudoargumentación' [17], es decir, aducen argumentos que no contribuyen al diálogo crítico, sino que lo entorpecen. Para Maliandi, 'el diálogo crítico sólo es posible cuando los participantes están, en efecto, dispuestos a modificar, eventualmente, sus propias opiniones, y a subordinar sus propios intereses al acuerdo intersubjetivo'. Por tanto, las capacidades argumentativas aparecen estrechamente ligadas a la capacidad intersubjetiva de cada hablante o, en otras palabras, a su grado de desarrollo de teoría de la mente; argumentar exige la capacidad de tener en cuenta el punto de vista ajeno, la posible refutación del interlocutor.

\section{Conclusiones}

- Ni en la edad infantil ni en la adolescente se encuentran diferencias radicales, cualitativas, respecto a los textos elaborados por el grupo control. Hay diferencias de grado, no de uso.

- Mientras que en la edad infantil el grupo control utilizaba más argumentos por texto (5,5 frente a $3,3)$, en la adolescencia vemos que los hablantes
Tabla I. Evolución argumentativa infancia-adolescencia.

\begin{tabular}{lcc}
\hline & Infancia & Adolescencia \\
\hline Argumentos por texto & 3,3 & 5,5 \\
\hline Instrucción & 28,2 & 32,9 \\
\hline Sanción & 33,3 & 12,7 \\
\hline Altruista & 28,3 & 25,9 \\
\hline Falaz & 10,3 & 22,8 \\
\hline
\end{tabular}

Tabla II. Estrategias argumentativas en adolescentes con TDAH y del grupo control.

\begin{tabular}{lcc}
\hline & Control & TDAH \\
\hline Argumentos por texto & 3,0 & 5,5 \\
\hline Instrucción & 30,9 & 32,9 \\
\hline Sanción & 6,3 & 12,7 \\
\hline Altruista & 23,8 & 25,9 \\
\hline Tópica & 12,7 & 10,9 \\
\hline Falaz & 25,9 & 17,1 \\
\hline
\end{tabular}

con TDAH aducen un mayor número de justificaciones para cada tesis.

- En ambas franjas de edad, el grupo con TDAH utiliza doblemente las estrategias de sanción, muy especialmente las de sanción negativa. El grupo de control recurre a este tipo de argumentos en una proporción del $17,7 \%$ (infancia) y el $6,3 \%$ (adolescencia), mientras que el grupo con TDAH utiliza este argumento en una proporción del 33,3\% y el 12,7\%, respectivamente. Interpretamos esta diferencia como discurso ecoico de los mensajes adultos.

- Inversamente, en el grupo control se recurre más a estrategias falaces (tópicas, tautológicas, circulares, de autoridad).

- En ambas franjas de edad se utiliza similarmente la argumentación lógica, razonable: un 38,4\% y 30,9\% de los argumentos del grupo control, y un 28,2\% y $32,9 \%$ de los argumentos del grupo con TDAH. 
Bibliografía

1. Bruner JS. Realidad mental y mundos posibles. Barcelona: Gedisa; 1988.

2. Bruner JS. Explaining and interpreting: two ways of using mind. In Harman G, ed. Conceptions of the human mind. Hillsdale: Lawrence Erlbaum; 1993. p. 123-36.

3. Gallardo-Paúls B. Pragmática para logopedas. Cádiz: Universidad de Cádiz; 2007.

4. Gallardo-Paúls B. Las huellas lingüísticas de teoría de la mente: intersubjetividad y enunciación. Rev Neurol 2008; 46 (Supl 1) S29-35.

5. Gallardo-Paúls B. Valoración del componente pragmático a partir de datos orales. Rev Neurol 2009; 48 (Supl 2): S57-61.

6. Gimeno M. Adolescencia y argumentación. 2nd International Clinical Linguistics Conference, Madrid, noviembre de 2009.

7. Ntirampeba P. La progressions en didactique du texte argumentatif écrit. Revue Canadienne de Linguistique Appliquée 2003; 6: 159-69.

8. Brassart DG. Does a prototypical argumentative schema exist? Argumentation 1996; 10: 163-74.

9. Pardos A, Fernández-Jaén A, Martín Fernández-Mayoralas D. Habilidades sociales en el trastorno por déficit de atención/ hiperactividad. Rev Neurol 2009; 48 (Supl 2): S107-11.

10. López-Villalobos JA, Serrano-Pintado I, Delgado SánchezMateos J, Ruiz-Sanz F, Sánchez-Azón MI, Sacristán-Martín
AM. Trastorno por déficit de atención/hiperactividad: perfil intelectual y factor de independencia a la distracción. Rev Neurol 2007; 44: 589-95.

11. Soriano M. Intervención cognitivo-conductual en niños con TDAH. XXVI Congreso AELFA, La Laguna, julio de 2008.

12. Mulas F, Etchepareborda MC, Abad-Mas L, Díaz-Lucero A, Hernández S, De la Osa-Langreo A, et al. Trastornos neuropsicológicos de los adolescentes afectos de trastorno por déficit de atención con hiperactividad. Rev Neurol 2006; 43 (Supl 1): S71-81.

13. Barkley RA. Niños hiperactivos. Cómo comprender y atender sus necesidades especiales. Barcelona: Paidós; 2002.

14. Barkley RA, Murphy KR, Dupaul GG, Bush T. Driving in young adults with attention deficit hyperactivity disorder: knowledge, performance, adverse outcomes, and the role of executive functioning. J Int Neuropsychol Soc 2002; 8: 655-72.

15. Mulas F, Mattos L, De la Osa-Langreo A, Gandía R. Trastorno por déficit de atención/hiperactividad: a favor del origen orgánico. Rev Neurol 2007; 44 (Supl 3): S47-9.

16. Servera M. Modelo de autorregulación de Barkley aplicado al trastorno por déficit de atención con hiperactividad: una revisión. Rev Neurol 2005; 40: 358-68.

17. Padilla C, Carlino P. Alfabetización académica e investigación acción. In Parodi G, ed. Alfabetización académica y profesional en el siglo XxI: leer y escribir desde las disciplinas. Santiago de Chile: Planeta; 2010. p. 36-59.

\section{Textual pragmatics in adolescents with attention deficit hyperactivity disorder: argument}

Introduction. Clinical linguistics involves a study of linguistic deficits which focuses on a series of aspects that range from strictly formal, grammatical points to the effective and contextualised use of language. Thus, it is also inevitably concerned with the cognitive, i.e. mental, correlate of such language use, whose basic textual dimensions are narration and argument.

Aims. To describe the argumentative skills in adolescents with attention deficit hyperactivity disorder (ADHD) and to examine their relationship with academic achievement and sociability.

Materials and methods. We analysed 79 argumentative texts written by adolescents with ADHD, using a methodology from cognitive linguistics and from theories of argumentation with a dialogical foundation.

Results and conclusions. Adolescents with ADHD provided a greater number of arguments than those in the control group, but with a higher predominance of emotional and negative sanction strategies compared with a greater use of fallacious or circular arguments in those in the control group; the difference between the use of rational arguments in the two groups is not significant.

Key words. ADHD. Linguistic deficit. Textual pragmatics. 